\title{
Potential of space-based infrared Bracewell interferometers for planet detection
}

\author{
D. Defrère*a ${ }^{* a}$ O. Absil ${ }^{b}$, C. Hanot ${ }^{a}$ and M. Fridlund ${ }^{c}$ \\ ${ }^{a}$ Institut d'Astrophysique et de Géophysique de Liège (IAGL), Université de Liège, Belgium \\ ${ }^{b}$ Laboratoire d'Astrophysique de l'Observatoire de Grenoble, France \\ ${ }^{c}$ Astrophysics Mission Division, ESA (ESTEC), The Netherlands
}

\begin{abstract}
The Darwin and TPF-I space missions will be able to study the atmosphere of distant worlds similar to the Earth. Flying these space-based interferometers will however be an extraordinary technological challenge and a first step could be taken by a smaller mission. Several proposals have already been made in this context, using the simplest nulling scheme composed of two collectors, i.e., the original Bracewell interferometer. Two of these projects, viz. Pegase and the Fourier-Kelvin Space Interferometer, show very good perspectives for the characterisation of hot extra-solar giant planets (i.e., Jupiter-size planets orbiting close to their parent star). In this paper, we build on these concepts and try to optimise a Bracewell interferometer for the detection of Earth-like planets. The major challenge is to efficiently subtract the emission of the exo-zodiacal cloud which cannot be suppressed by classical phase chopping techniques as in the case of multi-telescopes nulling interferometers. We investigate the potential performance of split-pupil configurations with phase chopping and of OPD modulation techniques, which are good candidates for such a mitigation. Finally, we give a general overview of the performance to be expected from space-based Bracewell interferometers for the detection of extra-solar planets. In particular, the prospects for known extra-solar planets are presented.
\end{abstract}

Keywords: Nulling interferometry, space-based mission, extra-solar planets.

\section{INTRODUCTION}

Nulling interferometry is one of the most promising techniques to study extra-solar planets. The simplest way to implement it is to use a Bracewell interferometer. ${ }^{1}$ The principle is to combine the beams coming from two telescopes in phase opposition so that a dark fringe appears on the line of sight, which strongly reduces the starlight emission while letting through the light from an off-axis source by adjusting the baseline length. This concept is used in several space mission projects, like Pegase ${ }^{2}$ and the Fourier-Kelvin Stellar Interferometer, ${ }^{3}$ aiming at studying extra-solar giant planets (EGP) located close to their parent star. The detection of Earth-like planets is much more challenging due to the higher star/planet contrast. It requires a higher nulling stability over a longer period and specific methods of modulation to suppress the emissions of the local and exo-zodiacal clouds. This should be achieved by the Darwin ${ }^{4}$ and TPF- $\mathrm{I}^{5}$ projects, which are based on a more complex scheme of nulling using at least three collectors. These missions will achieve direct detection and atmospheric characterization of Earth-like planets orbiting in the habitable zone (HZ) of nearby main sequence stars (within $\sim 30 \mathrm{pc}$ ). They will be fantastic tools to search for biological signatures in our vicinity. ${ }^{6}$ Beyond these ambitious objectives, the scientific goals of these missions also include the origin of the planetary systems, its evolutionary history and its future development. The study of hot EGP with Pegase or the Fourier-Kelvin Space Interferometer (FKSI) would already be very fruitful in that respect, but it would be interesting to know what could be achieved with a more advanced Bracewell concept. Improving the specifications of these missions, such as the size of the telescopes, could increase the sensitivity and give additional information on smaller planets. The goal of this study is to assess the performance of such a mission.

\footnotetext{
* E-mail: defrere@astro.ulg.ac.be, Telephone: 003243669713
} 


\section{INSTRUMENTAL CONCEPT}

\subsection{Overview of the Pegase design}

This study considers the CNES formation flying demonstrator Pegase as a baseline. ${ }^{2}$ Pegase consists in a two 40-cm diameter apertures interferometer observing in the near-infrared $(2.5-5 \mu \mathrm{m})$ and formed of three free flying spacecrafts orbiting at the Lagrange point L2. Thanks to this location, the spacecrafts and the focal plane assembly can be passively cooled down to respectively $90 \pm 1 \mathrm{~K}$ and $55 \pm 0.1 \mathrm{~K}$. Visibility measurements and recombination in nulling mode are both possible with a spectral resolution of about 60 . The formation flying constraints allow baseline lengths from $40 \mathrm{~m}$ to $500 \mathrm{~m}$ giving an angular resolution in the range of 0.5 mas to 30 mas. The fine-tuning of the optical path difference (OPD in the following) is performed by a dedicated control loop based on a fringe sensor measuring the observed central target in the 0.8-1.5 $\mu \mathrm{m}$ range and an optical delay line. The intensity control is performed by a fine pointing loop using a field relative angle sensor operating in the 0.6-0.8 $\mu \mathrm{m}$ range. This implementation allows to control the OPD down to a level of $2.5 \mathrm{~nm} \mathrm{rms}$ and the tip/tilt errors to a value of 30 mas rms, corresponding to a nulling ratio of $10^{-4}$ stable at the level of $10^{-5} .^{2}$

\subsection{Modifying Pegase}

If Pegase was pointed towards a Solar system located at 20 pc, it would be inefficient for Earth-like planet detection for several reasons. The main show-stopper is the starlight cancellation which is not sufficiently stable and requires a better control of the OPD. Of minor relevance when observing hot EGPs, the local and exo-zodiacal clouds become very problematic for Earth-like planets and will be major sources of noise in the final budget. A way to improve the sensitivity of the instrument is to optimise the wavelength range of observation. Since Earth-like planets are cooler than hot EGPs, their maximum brightness is shifted toward longer wavelengths. For example, at $10 \mu \mathrm{m}$, the emission of an Earth is about 30 times larger than at $2.5 \mu \mathrm{m}$ while the flux of the exo-zodiacal cloud increases only by a factor 4 . In addition, the stellar flux decreases and is 10 times lower at 10 $\mu \mathrm{m}$ than at $2.5 \mu \mathrm{m}$. Nevertheless, increasing the observation wavelength is limited by the thermal emission of the instrument which becomes dominant at long wavelengths. The thermal specifications of Pegase would cause overwhelming background fluctuations for Earth-like planets detection. To reach this objective, the instrument has to be cooled down and more thermally stable. This is part of the optimisation presented in this study. The parameters used in our simulations are listed in Table 1.

\begin{tabular}{ll}
\hline Spectral & \\
Wavelength & $10 \mu \mathrm{m}$ \\
Resolution & 60 \\
& \\
Target system & $20 \mathrm{pc}$ \\
Distance & $1 \mathrm{R}_{\mathrm{Sun}}$ \\
Star radius & $5770 \mathrm{~K}$ \\
Star eff. temperature & $0.56 \mathrm{Jy}$ \\
Star flux & $1 \mathrm{R}$ Earth \\
Planet radius & $50 \mathrm{mas}$ \\
Planet angular offset & $265 \mathrm{~K}$ \\
Planet eff. temperature & $6.00 \mathrm{E}-8 \mathrm{Jy}$ \\
Planet flux & $1 \mathrm{solar}$ system zodi \\
Exo-zodi dust & $1.13 \mathrm{E}+7 \mathrm{Jy} / \mathrm{sr}$ \\
Local zodi brightness & \\
& \\
Bracewell specifications & $1 \mathrm{~m}$ \\
Telescope diameter & $20 \mathrm{~m}$ \\
Baseline length & $7 \%$ \\
Throughput &
\end{tabular}

Table 1. Parameters used in this study
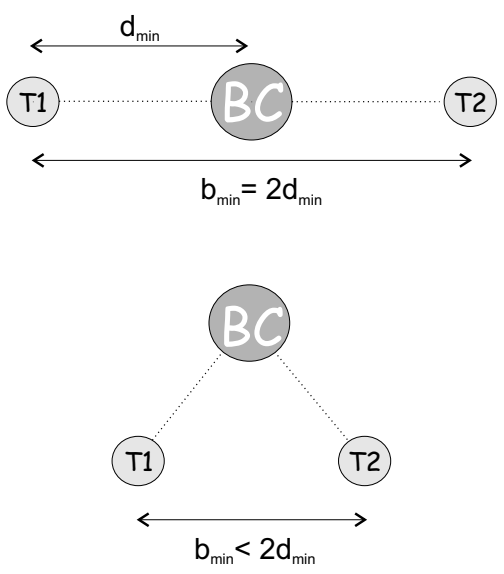

Figure 1. Above: nominal linear flying configuration of Pegase with a central beam combiner. Bottom: modified flying configuration of Pegase allowing a shorter interferometric baseline length. 
The observing scenario is based on the one of Pegase. Since the inclination and the position angle of the planetary orbit are unknown, three observations with baselines separated by $60^{\circ}$ will be obtained on each target. By consequence, the bright fringe will be closer than $30^{\circ}$ from the actual planet position angle for at least one observation and the planet flux will be reduced by a maximum of $5 \%$ in the worst case. In addition, since the ephemeris of the planet is unknown, 3 different sets of observations along the orbit are necessary to reach a high level of confidence in case of non detection. A duty cycle of $50 \%$ is also considered.

Finally, assuming a minimum anti-collision baseline length of $20 \mathrm{~m}\left(\mathrm{~d}_{\min }\right)$, the linear configuration of Pegase with a central beam combiner is not convenient to survey the habitable zone of most $\mathrm{F}$ and $\mathrm{G}$ stars of the Darwin catalogue ${ }^{7,8}$ which require baselines shorter than $40 \mathrm{~m}$ at $10 \mu \mathrm{m}$. To solve this problem, a triangular flying configuration is considered, with the beam combiner spacecraft at equal distance from collector spacecrafts (see Figure 1).

\section{SIGNALS AND NOISES IN SPACED-BASED INTERFEROMETRY}

\subsection{Instrumental stellar leakage}

In nulling interferometry, a key quantity is the null depth which determines the level of on-axis stellar rejection. This null depth undergoes fluctuations due to instrumental imperfections such as co-phasing errors, wavefront errors or mismatches in the intensities of the beams. The influence of all these contributions is called instrumental leakage, which acts both as bias, by introducing an additional amount of unwanted signal in the nulled output, and as a noise through its instability. Assuming that the instrument behaves in the same way during the three successive observations of the same system, the average instrumental stellar leakage can be calibrated and only instability noise (including long-term drifts) and shot noise associated to the mean stellar leakage have to be considered in the total noise budget.

\section{Leakage due to the OPD}

Since the actual power spectral densities of instrumental perturbations are not available, the estimation of instability noise will be made assuming that the rms leakage is equal to its mean value. This assumption is supported by the results obtained on the GENIE and ALADDIN studies. ${ }^{9,10}$ The contribution of the residual OPD error to the stellar leakage can be estimated using the following formula: ${ }^{11}$

$$
\sigma_{\mathrm{OPD}} \leq \frac{\lambda}{\pi} \sqrt{N_{i}}
$$

where $N_{i}$ is the required stability for the instrumental nulling ratio. This means that a stability at the level of $\sigma_{\mathrm{OPD}} \leq 1 \mathrm{~nm}$ is required to cancel the star emission by a factor of $10^{7}$ at an observing wavelength of $10 \mu \mathrm{m}$. Very encouraging results have been obtained recently with the adaptive nuller ${ }^{12}$ at Jet Propulsion Laboratory with the demonstration of a broadband null of $1.2 \times 10^{-5}$ over a $32 \%$ bandwidth centered in the 8-12 $\mu \mathrm{m}$ band*. Reaching a stability of $1 \mathrm{~nm}$ at $10 \mu \mathrm{m}$ is still challenging but should be realistic in the near-term future. ${ }^{13}$ This value will be considered in this study. Note however that Eq. 1 is a first order estimation and does not take into account the non-linear second order error terms which are expected to dominate the instrumental leakage. ${ }^{14}$

\section{Leakage due to tip/tilt errors}

The other major contributors to the stellar leakage are the wavefront errors and the intensity mismatch between the beams. Because modal filtering is used, wave front errors will be traded against intensity errors which are less severe source of instrumental leakage and add to the true intensity mismatch. In fact, true intensity errors are not expected to be a significant source of intensity mismatch and will be neglected in the following discussion. The instrumental nulling ratio $N_{i}$ due to the tip-tilt error can be estimated with the following formula, taking into account the modal filtering process: ${ }^{15}$

$$
N_{i}=\frac{\left(\pi D \sigma_{\theta}\right)^{4}}{64 \lambda^{4}}
$$

\footnotetext{
${ }^{*}$ see the adaptive nuller website: http://planetquest.jpl.nasa.gov/TPF-I/adaptiveNullerTestbed.cfm
} 
where $D$ is the telescope diameter and $\sigma_{\theta}$ the residual tip/tilt error. The tip-tilt control device which will be implemented in Pegase should reach a typical residual of 30 mas rms. ${ }^{2}$ At $10 \mu \mathrm{m}$ and for 1 -m size telescopes, a tip/tilt residual below 22 mas rms is necessary to get an SNR of 5 for an Earth located at 20 pc. The use of larger telescopes in combination with a two-stage control loop implementation will improve the pointing accuracy with respect to the Pegase nominal value (30 mas $\mathrm{rms}$ ). Without an appropriate system study, it is difficult to give an accurate estimation of this value but a tip/tilt residual of 20 mas rms should not be too optimistic and will be assumed in this study.

\subsection{Geometric stellar leakage}

Because of the finite size of the target star, a residual stellar flux appears in the nulled output. This deterministic part of the stellar leakage is called the geometric leakage and is given by: ${ }^{10}$

$$
N=\frac{\pi^{2}}{4}\left(\frac{b \theta_{\star}}{\lambda}\right)^{2}
$$

where $b$ is the baseline length and $\theta_{\star}$ the stellar angular radius. At $10 \mu \mathrm{m}$, the contribution of geometric leakage for a Sun-like star located at 20 pc is about 125 times larger than the flux of the planet in the nulled output. To reveal the hypothetic planetary companion, the most straightforward method relies on the knowledge of the target star angular radius, which is the only unknown parameter in Eq. 3. The angular radii of Darwin target stars can be determined with a typical accuracy of $1 \%$ which gives a precision of $2 \%$ on the estimation of the stellar leakage. ${ }^{16}$ This level of calibration is however not sufficient to get a SNR of 5 for an Earth located at 20 pc.

\subsection{The thermal background and its fluctuations}

\section{Exo-zodiacal dust emission}

An exo-zodiacal cloud similar to the local zodiacal disk is much brighter than an Earth-like planet with an integrated flux about 400 times larger at $10 \mu \mathrm{m}$. Filtered through the interferometer transmission map, the residual contribution does not exceed $30 \%$ of the total flux for a disk located at 20 pc. In addition, since several observations of the same system will be performed, the morphology of the dust cloud can be constrained and model-fitted. Taking into account both contributions, a conservative value of $10 \%$ is assumed in this study for the residual exo-zodiacal emission. To go further, fast modulation techniques have been developed in the context of Darwin/TPF but they require the use of at least 3 collectors. ${ }^{1,17}$ More specific methods such as the split-pupil configuration and the OPD modulation have to be used for a single Bracewell. ${ }^{18,19}$ These good candidates are reviewed by Hanot $^{20}$ and briefly discussed in section 5.3 .

\section{Local zodiacal dust emission}

The local zodiacal dust cloud produces a foreground through which the interferometer will observe. Its thermal emission is estimated by the zodiacal disk model of Kelsall. ${ }^{21}$ The model takes into account the dust in the line of sight of the interferometer located at the Lagrange point L2 and pointing in the anti solar direction. At 10 $\mu \mathrm{m}$, the total background emission associated with the local zodiacal disk is about $2 \times 10^{-4}$ times larger than the flux coming from an Earth at $20 \mathrm{pc}$ and received by an 1-m telescope. This emission presents a slow variation with typical periods of $13,27,36,47,52$, and 72 days $^{21}$ which are very problematic for long integration times. However, for integration times of the order of some hours, the variation of this emission can be considered as negligible ${ }^{22}$ and remains below the residual contribution of the exo-zodiacal cloud.

\section{Instrumental background}

The thermal emission of the instrument is modelled as a grey body emission, i.e. a black-body emission multiplied by a constant emissivity factor related to the optical train. The mean value of the instrumental background can be removed with the three inclined observations and only the fluctuations remain. In order not to induce significant variations of the background emission, a high thermal stability is therefore required. To detect at $10 \mu \mathrm{m}$ an Earth located at $20 \mathrm{pc}$ (SNR of 5), the thermal stability has to be as low as $0.1 \mathrm{~K}$ rms for an instrument cool down to $45 \mathrm{~K}$. 


\section{ANALYSIS FOR AN EARTH AT 20 PC}

Following the previous discussion, Table 2 shows the different requirements on a 1-m Bracewell to achieve at 10 $\mu \mathrm{m}$ an SNR of 5 when observing an Earth located at $20 \mathrm{pc}$. It also shows the specifications considered for this study and the minimum size of the planet (located in the middle of the HZ) detectable with it.

\begin{tabular}{lcc|c} 
& Req. & Spec. & $\mathrm{R}_{\text {Earth }}$ \\
\hline OPD [nm] & 0.47 & 1 & 2.1 \\
Tip/tilt [mas] & 22 & 20 & 0.8 \\
Stellar radius knowledge [\%] & 0.8 & 1 & 3.6 \\
Exo-zodiacal subtraction [\%] & 0.05 & 10 & 11.2 \\
Instrument temperature [K] & 45.3 & 45 & 1 \\
Integration time [days] & 3000 & & \\
$\quad$ 20 surveys/year [h] & - & 24 & 5.7 \\
$\quad 50$ surveys/year [h] & - & 10 & 7.2 \\
100 surveys/year [h] & - & 5 & 8.5 \\
\hline
\end{tabular}

Table 2. First column: requirements on an 1-m Bracewell to detect an Earth-like planet located at $20 \mathrm{pc}$ at $10 \mu \mathrm{m}$. Second column: specifications of the 1-m Bracewell considered in this study. Third column: minimum radius of the planet detectable with it. The shot and detector noises impose a lower limit on the integration time and the three last rows give the minimum radius of the planet detectable in 5, 10 or 24 hours (corresponding respectively to 100,50 or 20 systems surveyed per year).

Due to the limited suppression of the exo-zodiacal emission, a 1-m aperture single Bracewell could not detect planets smaller than Jupiter (about $11 \mathrm{R}_{\text {Earth }}$ ) and orbiting in the middle of the HZ of a Sun located at $20 \mathrm{pc}$. With an appropriate method to subtract the exo-zodiacal emission, 7 Earth-radii planets would be possible to detect in 10 hours which corresponds to 50 systems surveyed per year. In 24 hours, the detection of 6 Earth-radii planets could be achieved and potentially 4 Earth-radii planets for a longer integration time. The reason is that the limitation coming from the integration time is due to the shot and detector noises which can be minimized by a sufficiently long integration time as shown by Eq. 4, giving the SNR per spectral channel:

$$
\mathrm{SNR}_{n}=\frac{F_{p} \cdot t}{\sqrt{\left(N_{s}^{2}+N_{d}^{2}\right) t}+\left(\sigma_{i}+\sigma_{c}+\sigma_{b}\right) t},
$$

where $F_{p}$ is the planet's flux, $N_{s}$ the shot noise, $N_{d}$ the detector noise, $\sigma_{i}$ the instrumental nulling noise, $\sigma_{c}$ the instrumental calibration noise, $\sigma_{b}$ the background fluctuation noise and $t$ the integration time. Shot noise is dominated mainly by the stellar leakage and the contribution from the local zodiacal cloud. The detection unit features a Si:As focal plane array with a read out noise assumed to amount to 10 electrons per pixel. Dark current is supposed to be negligible by cooling down the detector to an appropriate temperature (a few K). The SNR per spectral element is represented by the solid line in Figure 2 with respect to the wavelength.

Figure 2 shows that the optimum wavelength is effectively near $10 \mu \mathrm{m}$ and that the exo-zodiacal emission is dominant in the 5-12 $\mu \mathrm{m}$ band. In order to improve the detection capability, one can combine the spectral channels, using Eq. 5: ${ }^{14}$

$$
\mathrm{SNR}_{\text {tot }}=\left[\sum_{n_{\text {indep }}} \mathrm{SNR}_{n}^{2}\right]^{1 / 2},
$$

where the sum is performed over the noise independent spectral channels. Determining their number would require a full analysis and is beyond the scope of this paper (some clues are given in the paper of Lay ${ }^{14}$ ). For the time being, with a spectral resolution of 60 , the spectral channels are narrow at $10 \mu \mathrm{m}$ (about $0.16 \mu \mathrm{m}$ wide) and we will assume than only $10 \%$ of the channels are noise independent (about $1.6 \mu \mathrm{m}$ wide). The percentage of noise independent spectral channels is denoted $\eta$ in the following. 


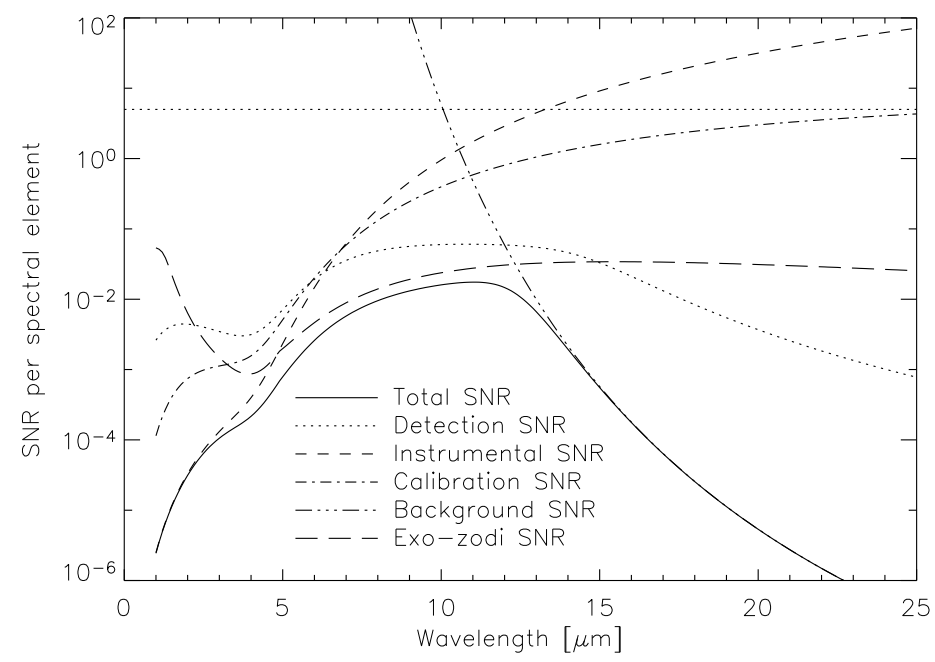

Figure 2. Individual SNRs contributing to the total SNR (solid line) for an Earth-like planet located at 20pc with respect to the wavelength, after 10 hours of integration and 1-m diameter collectors.

\section{DETECTION THRESHOLD}

\subsection{Planet signal}

In good approximation, the flux emitted by an extra-solar planet and detected by an Earth-based observer writes: ${ }^{16}$

$$
F_{p}(\lambda)=A_{b} \frac{\pi R_{\star}^{2}}{d^{2}} B_{\lambda}\left(T_{\star}\right) \Phi(\Theta) \frac{R_{p}^{2}}{4 a^{2}}+\frac{\pi R_{p}^{2}}{d^{2}} f(\Theta) B_{\lambda}\left(T_{p}\right),
$$

where $B_{\lambda}(T)$ represents the surface brightness per wavelength unit for a blackbody at temperature $T, d$ is the distance to the target star, $a$ the semi-major axis of the planetary orbit and $R_{p}$ the planetary radius. $\Phi(\Theta)$ and $f(\Theta)$ are the phase function affecting respectively the reflected and the emitted emissions. $A_{b}$ is the Bond albedo, defined as the ratio between the total reflected and total incident powers. The first term in Eq. 6 represents the reflected part of the stellar spectrum while the second term represents the thermal emission of the planet. $T_{p}$ is the equilibrium temperature of the planet and can be calculated by the following formula: ${ }^{16}$

$$
T_{p}=T_{\star}\left(1-A_{b}\right)^{1 / 4}\left(\frac{R_{\star}}{2 a}\right)^{1 / 2} .
$$

The Bond albedo used to compute the equilibrium temperature depends on several parameters such as the chemical composition of the planet's atmosphere and surface. The results presented in this paper assume a Bond albedo of 0.306 for rocky planets by analogy to the Earth's albedo, although the surface and the potential atmosphere are probably not the same. This value has also the advantage to be intermediate among the Bond albedos of the Solar system rocky planets ( 0.119 for Mercur and 0.750 for Venus). For the giants planets, a value of 0.10 is assumed as derived by Baraffe ${ }^{23}$ for hot EGPs instead of Jupiter's classical value of 0.342 , since the regions close to the star will mostly be investigated. Finally, following the simulations of core accretion reported by Ida, ${ }^{24}$ the limit between gaseous and rocky planets will be considered around 15 Earth masses.

\subsection{Results for a Sun at 20 pc}

The purpose of this section is to extend the analysis presented in the previous sections by considering planets with different orbits. This general approach requires numerical simulations which have been done by adapting a top-level performance prediction tool developed by O. Absil for Pegase ${ }^{16}$ and using several routines from the Darwin science simulator, DARWINsim. ${ }^{25} \mathrm{~A}$ crucial parameter in the performance assessment is the distance 
between the planet and its parent star. In accordance with Eq. 6 and Eq. 7, the closer the planet is to its parent star the brighter it is, which improves also the contrast with the background sources. The optimum wavelength is also modified since the planet becomes hotter. Optimizing the wavelength in agreement with the semi-major axis of the planet's orbit, the detection threshold, i.e. the minimum size of the planet detectable, is represented in Figure 3 for a Sun-like star located at 20 pc (the detection threshold for detection is set at a global SNR of 5). For practical reasons (it is difficult to cover the full wavelength range with one set of glasses and single-mode spatial filters), the width of the total wavelength band has been set to $4 \mu \mathrm{m}$.

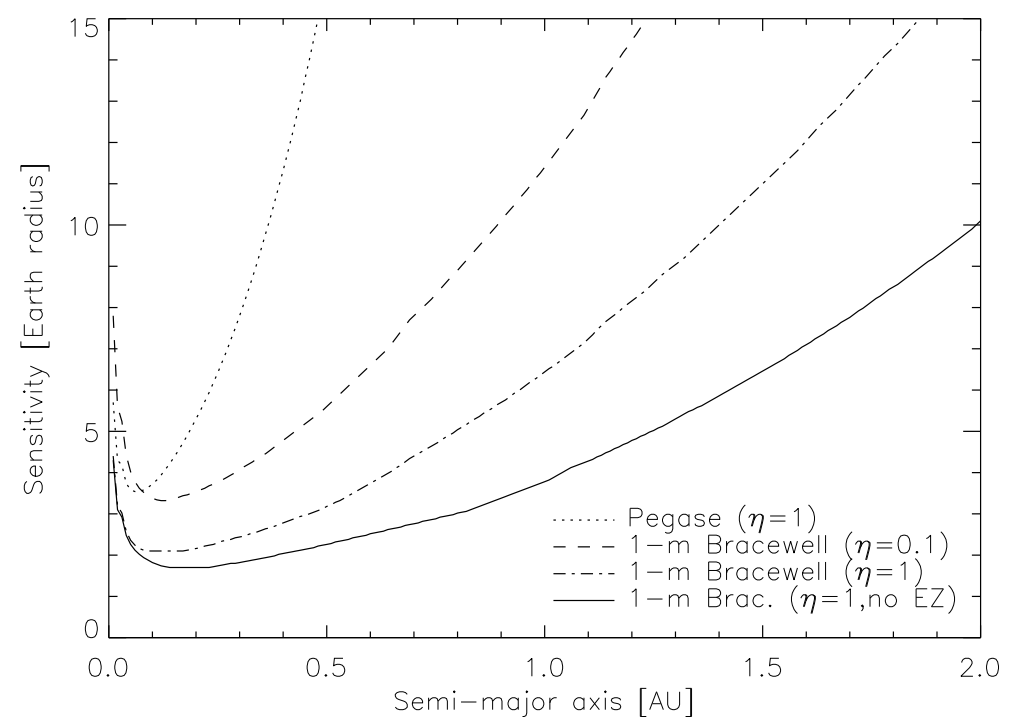

Figure 3. Sensitivity of Pegase (dotted), 1-m Bracewell with respectively $10 \%$ (dashed) and 100\% (dash-dot) independent spectral channels and without exo-zodiacal cloud (solid) for 10 hours integration time and observing a Sun-like star located at 20 pc. For each semi-major axis, the sensitivity is computed in the optimum $4-\mu$ m wide wavelength band.

Each curve represents the detection threshold for an integration time of 10 hours (about 50 surveys per year). The dotted curve shows the sensitivity domain of Pegase assuming a perfect combination of the spectral channels $(\eta=1)$ in the 2.5-5 $\mu \mathrm{m}$ wavelength range. The best sensitivity arises for planets located very close to the star with 4 Earth-radii planets detectable at about $0.05 \mathrm{AU}$ and Jupiter-size planets detectable till 0.4 AU. Including the exo-zodiacal emission, the dash-dot curve shows the improvement of the capabilities by considering a $1-\mathrm{m}$ Bracewell working at $10 \mu \mathrm{m}$. Jupiter-size planets are detectable till about 1.6 AU while in the middle of the HZ, Neptune-size planets could be detected. Rocky planets are detectable in the close vicinity of the star $(\sim$ $0.1 \mathrm{AU})$. Going further, the solid curve shows the potential of the concept on the basis of 10 hours integration time, assuming a perfect subtraction of the exo-zodiacal emission in addition to the perfect combination of the spectral channels $(\eta=1)$. Small Neptune-like planets orbiting around a Sun located at $20 \mathrm{pc}$ are detectable within 10 hours in the middle of the HZ. Shot noise is now the limiting factor and a longer integration time is required to improve the sensitivity.

Finally, a more realistic assessment of the capabilities is given by the dashed curve, plotted considering that only $10 \%$ of the spectral channels are independent. This curve gives also an approximation of the minimum size of planets for which spectroscopy can be performed. As expected from the previous section, the performance would be limited to Jupiter-size planets in the middle of the HZ.

\subsection{Removing the exo-zodiacal emission}

As shown by Table 2, the residual of the exo-zodiacal emission is the main limiting factor. The level of performance could be considerably improved (4 Earth-radii instead of Jupiter-size planets detectable) by removing it from the nulled output. The basic method to modulate the planet signal against the exo-zodiacal emission is to 
rotate the interferometer along the line of sight but this can generally not be performed sufficiently fast and the planet signal is easily corrupted by long term drifts. Fast modulation can be implemented with the split-pupil configuration ${ }^{18}$ or with OPD modulation. ${ }^{19}$ In the case of split-pupil, both telescopes are divided in two parts, creating two independent Bracewell interferometers. The outputs of these two interferometers are then combined with a time-varying phase shift which allows to remove all point-symmetric emissions (in the ideal case). The division of the pupil introduces however significant additional complexity. ${ }^{16}$ The principle of OPD modulation is to sweep back and forth the dark fringe of the transmission map by modulating the pathlength in one arm of the interferometer. ${ }^{19}$ In that manner, all centro-symmetric sources are modulated at twice the modulation frequency. If the baseline length is chosen such as the planet locates on a grey fringe, the planet signal will be modulated at the modulation frequency and can be retrieved by using synchronous coherent demodulation. In principle, OPD modulation could be implemented at little increase of the system complexity. For both methods, only $50 \%$ of the planet signal is generally retrieved which reduces the actual performance. In his master thesis, Hanot shows that the split-pupil technique leads to better SNRs than the OPD modulation but remains pessimistic about the ability of both methods to reveal Earth-like planets in presence of instrumental errors and typical asymmetries of the cloud. ${ }^{20}$ A full investigation of these techniques will be the object of an upcoming paper.

\subsection{Study of the habitable zone: application to the Darwin catalogue}

The application to the Darwin catalogue ${ }^{7,8}$ allows to extend the study to stars of different spectral types. The prime targets of the catalogue, i.e. the single star systems, amount to 628 stars $(43 \mathrm{~F}, 100 \mathrm{G}, 244 \mathrm{~K}$ and 241 $\mathrm{M}$ stars), in our close neighbouroud ( $\leq 25 \mathrm{pc}$ ). Around each of these stars, a planet is supposed to be present in the middle of the HZ. Expressed in AU, the position of the HZ is given in good approximation by the following formula: ${ }^{26}$

$$
r_{\mathrm{HZ}}=\left(\frac{T_{\star}}{T_{\odot}}\right)^{2} \frac{R_{\star}}{R_{\odot}}
$$

which corresponds to 2.3, 1.3, 0.7 and $0.2 \mathrm{AU}$ for F0V, G0V, K0V and M0V stars respectively. To estimate the performance of a 1-m Bracewell, the integration time required to reach an SNR of 5 for each target of the Darwin catalogue is computed. The SNRs are computed with the assumption that there is no exo-zodiacal emission so that the full potential of methods described in section 5.3 can be reached. The spectral channels are combined in the optimum 4- $\mu \mathrm{m}$ wide wavelength range with $\eta=1$. Note that according to Eq. 4 , only the part of the SNR due to the shot and detector noises can be improved by increasing the integration time. Targets for which $\sigma_{i}+\sigma_{c}+\sigma_{b}>5 F_{p}$ cannot reach an SNR of 5 and are thus not considered. The integration times of the remaining targets are then sorted by increasing values and added till the sum reaches the mission lifetime. The results are presented in Figure 4 which indicates the number of stars around which a planet of a given radius can be detected during the mission lifetime.

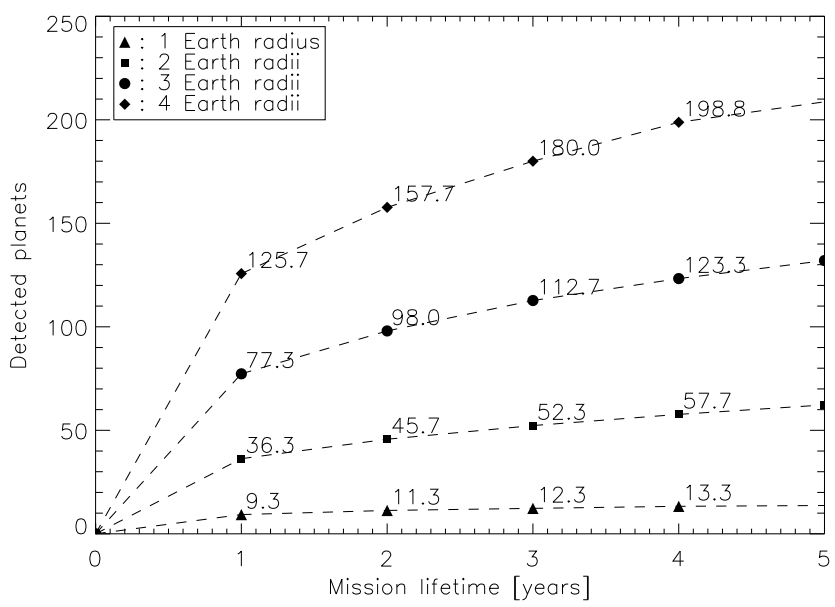

Figure 4. Number of stars around which a planet of a given radius can be detected with respect to the mission lifetime. 
During the first year of the mission, about 9 Earth-like or 36 two Earth-radii planets could be detected. A planet is considered detected when a total SNR of 5 is reached for at least one of the three observations for each position on the orbit. The gain by increasing the mission lifetime is not significative, particularly for the smallest planets. These detected planets are mainly around M stars as illustrated in Figure 5, showing the total integrated SNR for a rocky planet of 2 Earth radii and an integration time of 10 hours.

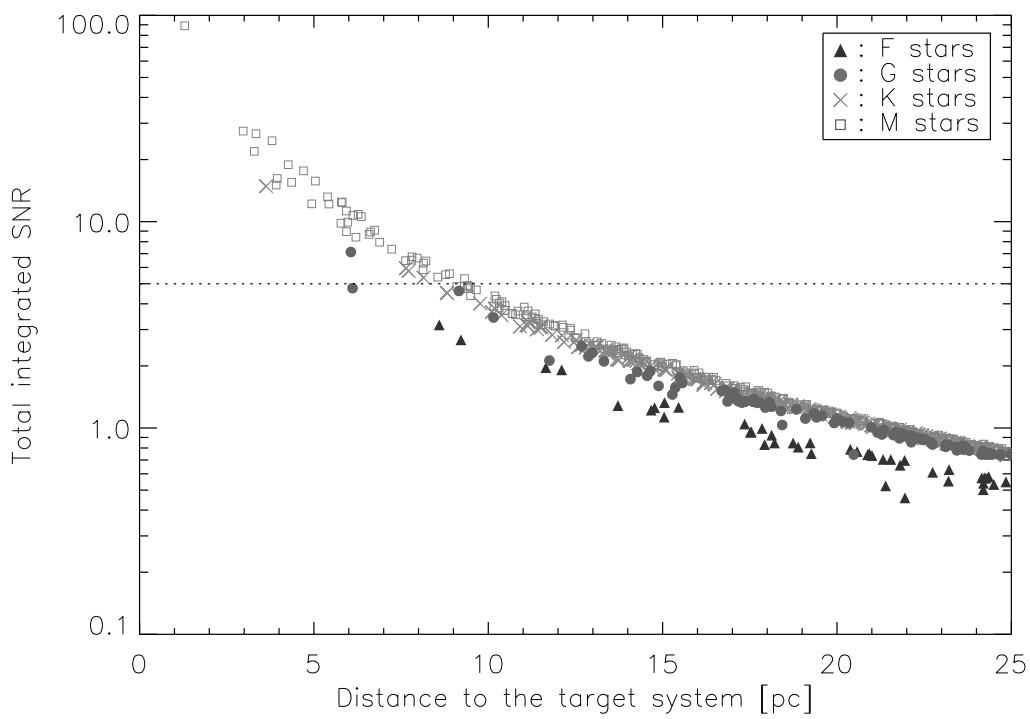

Figure 5. Total SNR for a two Earth-radii planet orbiting in the middle of the HZ of Darwin targets and for an integration time of 10 hours (assuming no exo-zodiacal cloud).

While an SNR of 5 is out of reach for F stars, all M-star planets within $\sim 10$ pc could be detected as well as several K-star and 1 G-star planets. M stars have been identified as relevant targets for future HZ planet search programs $^{27}$ and would therefore be very good candidates to survey. Note also that all 2 Earth-radii planets located beyond $10 \mathrm{pc}$ would not be detectable. In conclusion, the Bracewell interferometer presented in this study would be a convenient instrument to search $\mathrm{M}$ stars located within $10 \mathrm{pc}$ for planets of 2 Earth-radii. Some K and G stars might also be added to the target list.

\subsection{Application to known extra-solar planets}

Whereas 246 extra-solar planets have been discovered so far ${ }^{\dagger}$, only 61 of them are in our close vicinity (closer than $25 \mathrm{pc}$ ). Considering a sample of these 61 favorable targets, Table 3 gives the SNRs achievable by Pegase, the classic Bracewell with 1-m diameter telescopes including the noise contribution of the exo-zodiacal emission (1-m Bracewell) or not (1-m+ configuration), in the optimum spectral element and for an integration time of 10 hours. By definition, the 1-m+ configuration gives upper limits on the actual SNRs that we could expect from the split-pupil configuration and the OPD modulation technique.

Defining the good candidates for spectroscopy as those for which an SNR of 5 can be achieved in the optimum spectral element, Pegase could perform spectroscopy on 9 of the 61 targets (about 15\%), including 3 planets in the hot regime (closer than 0.1 AU from its parent star, see for instance $\tau$ Boo b and 51 Peg $b$ in Table 3) and 6 cooler planets like 70 Vir $b$ and 55 Cnc $b$. These results suggest that the Pegase target list could be extended to a significant number of planets outside the hot regime (see the master thesis of Defrère ${ }^{28}$ for the SNRs of the full target list).

\footnotetext{
${ }^{\dagger}$ see http://exoplanet.eu/catalog.php or http://exoplanets.org/ for an up-to-date list.
} 


\begin{tabular}{c|ccc|ccc|ccc}
\hline & \multicolumn{3}{|c|}{ Star } & \multicolumn{3}{c}{ Planet } & \multicolumn{3}{c}{ SNR } \\
\hline & $\begin{array}{c}\text { spectral } \\
\text { type }\end{array}$ & $\begin{array}{c}\text { dist. } \\
{[\mathrm{pc}]}\end{array}$ & $\begin{array}{c}\text { eclipt. } \\
\text { lat. }\end{array}$ & $\begin{array}{c}\text { S-maj. } \\
\text { axis }[\mathrm{AU}]\end{array}$ & $\begin{array}{c}\text { Min. mass } \\
{\left[\mathrm{M}_{\text {Jup }}\right]}\end{array}$ & $\begin{array}{c}\mathrm{T}_{\text {eq }} \\
{[\mathrm{K}]}\end{array}$ & Peg. & 1 -m & 1 -m+ \\
\hline Ups And b & F8V & 13.5 & 28.9 & 0.06 & 0.69 & 1570 & 2 & 3 & 4 \\
Ups And c & F8V & 13.5 & 28.9 & 0.83 & 1.98 & 420 & 2 & 18 & 41 \\
Ups And d & F8V & 13.5 & 28.9 & 2.53 & 3.95 & 240 & 0 & 4 & 14 \\
Tau Boo b & F7V & 15.6 & 26.5 & 0.05 & 3.90 & 1270 & 36 & 79 & 85 \\
55 Cnc b & G8V & 13.4 & 10.4 & 0.12 & 0.78 & 560 & 13 & 47 & 76 \\
55 Cnc c & G8V & 13.4 & 10.4 & 0.24 & 0.22 & 390 & 1 & 10 & 22 \\
55 Cnc d & G8V & 13.4 & 10.4 & 5.26 & 4.00 & 80 & 0 & 0 & 0 \\
55 Cnc e & G8V & 13.4 & 10.4 & 0.04 & 0.05 & 980 & 3 & 7 & 8 \\
70 Vir b & G5V & 18.1 & 21.3 & 0.48 & 7.44 & 500 & 6 & 39 & 59 \\
51 Peg b & G2V & 15.4 & 25.2 & 0.05 & 0.47 & 1290 & 5 & 8 & 9 \\
HD 69830 b & K0V & 12.6 & -0.55 & 0.08 & 0.03 & 830 & 2 & 4 & 5 \\
HD 69830 c & K0V & 12.6 & -0.55 & 0.19 & 0.04 & 540 & 1 & 5 & 7 \\
HD 69830 d & K0V & 12.6 & -0.55 & 0.63 & 0.06 & 290 & 0 & 2 & 3 \\
GI 581 b & M3 & 6.26 & 10.26 & 0.04 & 0.05 & 675 & 3 & 13 & 18 \\
GI 581 c & M3 & 6.26 & 10.26 & 0.07 & 0.02 & 510 & 0 & 4 & 7 \\
GI 581 d & M3 & 6.26 & 10.26 & 0.25 & 0.02 & 205 & 0 & 0 & 1 \\
Gliese 876 b & M4V & 4.7 & -6.6 & 0.21 & 1.93 & 220 & 0 & 18 & 47 \\
Gliese 876 c & M4V & 4.7 & -6.6 & 0.13 & 0.56 & 270 & 1 & 22 & 53 \\
Gliese 876 d & M4V & 4.7 & -6.6 & 0.02 & 0.02 & 680 & 3 & 7 & 9 \\
\hline
\end{tabular}

Table 3. Sample of known extra-solar planets located within $25 \mathrm{pc}$ from our solar system given with the SNRs achievable by Pegase, the classic 1-m Bracewell and the 1-m Bracewell without exo-zodiacal cloud (1-m+). The SNRs are given per spectral element at the optimum wavelength and for an integration time of 10 hours.

The use of the 1-m classic Bracewell allows to go up to 27 of the 61 targets (about 45\%), including 5 of the 9 remaining planets in the hot regime (see for instance 55 Cnc e, the hot-Neptune Gliese $876 d$ and GI $581 b$ in Table 3). Finally, with the 1-m+ configuration, spectroscopy can be performed on 38 targets, which represents about $60 \%$ of known extra-solar planets within 25 pc. The achievable SNRs can also be much better as illustrated by the Ups And system. More interesting, a SNR of 7 could be achieved in 10 hours on the recently discovered planet orbiting close to the HZ of its star, GI $581 \mathrm{c}$. Since the values given for the $1-\mathrm{m}+$ configuration are only upper limits, a realistic assessment of the capabilities of split-pupil/OPD modulation techniques would be the ability to do spectroscopy on 45 to $60 \%$ of all known extra-solar planets within 25 pc.

\section{SUMMARY AND CONCLUSION}

Infrared nulling interferometry is the core technique of several projects dedicated to the study of extra-solar planets. The most promising in terms of scientific return is undoubtedly the Darwin/TPF missions which should enable the spectroscopy of Earth-like planets in our close vicinity (within $30 \mathrm{pc}$ ). The achievement of this objective requires a complex scheme of nulling using at least 3 beam collectors. In this paper, we assess the capabilities of a simpler version of Darwin/TPF, which apart from consisting in only two 1-m diameter telescopes, presents Darwin-like specifications. The study is based on the infrared Bracewell interferometer Pegase, aimed at studying known hot Jupiters located within $150 \mathrm{pc}$. The main challenge to detect an Earth-like planet is the exo-zodiacal cloud which is 400 times brighter than an Earth located at $20 \mathrm{pc}($ at $10 \mu \mathrm{m})$. Specific methods to mitigate the exo-zodiacal emission in the case of Bracewell interferometers (viz. OPD modulation and splitpupil configuration) are addressed and results are given separately from those of the classic scheme (without the implementation of these methods).

Around a Sun located at $20 \mathrm{pc}$, the Bracewell interferometer presented in this study could detect in one year about 50 Neptune-like planets $\left(\mathrm{M} \sim 0.05 \mathrm{M}_{\text {Jup }}\right)$ orbiting in the middle of the habitable zone. The application to the Darwin catalogue has shown that smaller planets could be detected, with about 40 two Earth-radii planets detectable the first year of the mission. In particular, this "upgraded" Pegase or "down-scoped" Darwin would 
be a very efficient instrument to search $\mathrm{M}$ stars located within $10 \mathrm{pc}$ for planets of 2 Earth-radii. Finally, $45 \%$ of the 61 known extra-solar planets in the close vicinity of the solar system (within $25 \mathrm{pc}$ ) could be surveyed for spectroscopy instead of $15 \%$ with Pegase. This value reaches $60 \%$ if the exo-zodiacal emission is efficiently subtracted. These preliminary results need to be confirmed by detailed simulations, taking particularly into account the continuous rotation of the array and second order effects of the instability noise.

\section{ACKNOWLEDGMENTS}

The authors would like to thank R. den Hartog for his expert advice. Thanks also to J. Surdej, P. Riaud, D. Mawet and A. Magette for their contribution to the work presented in this paper. The first author acknowledges the financial support from the Belgian National Science Foundation ("FRIA").

\section{REFERENCES}

1. R. N. Bracewell, "Detecting nonsolar planets by spinning infrared interferometer," Nature 274, pp. 780-+, Aug. 1978.

2. J. M. Le Duigou, M. Ollivier, A. Léger, F. Cassaing, B. Sorrente, B. Fleury, G. Rousset, O. Absil, D. Mourard, Y. Rabbia, L. Escarrat, F. Malbet, D. Rouan, R. Clédassou, M. Delpech, P. Duchon, B. Meyssignac, P.-Y. Guidotti, and N. Gorius, "Pegase: a space-based nulling interferometer," in Space Telescopes and Instrumentation I: Optical, Infrared, and Millimeter. Edited by Mather, John C.; MacEwen, Howard A.; de Graauw, Mattheus W. M.. Proceedings of the SPIE, Volume 6265, pp. 62651M (2006)., 6265, July 2006.

3. R. K. Barry, W. C. Danchi, L. D. Deming, L. J. Richardson, M. J. Kuchner, S. Seager, B. J. Frey, A. J. Martino, K. A. Lee, M. Zuray, J. Rajagopal, T. T. Hyde, R. Millan-Gabete, J. D. Monnier, R. J. Allen, and W. A. Traub, "The Fourier-Kelvin stellar interferometer: a low-complexity low-cost space mission for high-resolution astronomy and direct exoplanet detection," Presented at the Society of Photo-Optical Instrumentation Engineers (SPIE) Conference 6265, July 2006.

4. C. V. M. Fridlund, L. d'Arcio, R. den Hartog, and A. Karlsson, "Status and recent progress of the Darwin mission in the Cosmic Vision program," in Advances in Stellar Interferometry. Edited by Monnier, John D.; Schöller, Markus; Danchi, William C.. Proceedings of the SPIE, Volume 6268, pp. 62680R (2006)., 6268, July 2006.

5. C. Beichman, P. Lawson, O. Lay, A. Ahmed, S. Unwin, and K. Johnston, "Status of the terrestrial planet finder interferometer (TPF-I)," in Advances in Stellar Interferometry. Edited by Monnier, John D.; Schöller, Markus; Danchi, William C.. Proceedings of the SPIE, Volume 6268, pp. 62680S (2006)., 6268, July 2006.

6. L. Kaltenegger, W. A. Traub, and K. W. Jucks, "Spectral Evolution of an Earth-like Planet," ApJ 658, pp. 598-616, Mar. 2007.

7. A. Stankov, "All sky target stars catalogue - no limit in ecliptic latitude," 2005.

8. L. Kaltenegger, C. Eiroa, A. Stankov, and M. Fridlund, "Nearby Stellar sample for the search for terrestrial planets. Target star catalogue for the Darwin Mission Study," A\&\&A, submitted, 2007.

9. O. Absil, V. Coudé du Foresto, M. Barillot, and M. Swain, "Nulling interferometry: comparison of performances between Antarctica and other ground-based sites," A\&A, submitted , 2007.

10. O. Absil, R. den Hartog, P. Gondoin, P. Fabry, R. Wilhelm, P. Gitton, and F. Puech, "Performance study of ground-based infrared Bracewell interferometers. Application to the detection of exozodiacal dust disks with GENIE," A\&A 448, pp. 787-800, Mar. 2006.

11. M. Ollivier, Contribution a la recherche d'exoplanetes coronographie interfrentielle pour la mission Darwin. PhD thesis, Universit Paris-Sud, Paris, France, 1999.

12. R. D. Peters, O. P. Lay, A. Hirai, and M. Jeganathan, "Adaptive nulling for the Terrestrial Planet Finder Interferometer," Presented at the Society of Photo-Optical Instrumentation Engineers (SPIE) Conference 6268, July 2006.

13. P. Lawson, "Results of Laboratory Experiments Kin Nulling Interferometry (1998-2006)," in the proceedings of the TPF/Darwin 2006 conference, 2006.

14. O. P. Lay, "Systematic Errors in Nulling Interferometers," Appl. Opt. 43, pp. 6100-6123, Nov. 2004. 
15. B. Mennesson, M. Ollivier, and C. Ruilier, "Use of single-mode waveguides to correct the optical defects of a nulling interferometer," Journal of the Optical Society of America A 19, pp. 596-602, Mar. 2002.

16. O. Absil, Astophysical studies of extrasolar planetary systems using infrared interferometric techniques. $\mathrm{PhD}$ thesis, Liège University, Liège, Belgium, 2006.

17. O. Absil, A. Karlsson, and L. Kaltenegger, "Inherent modulation: a fast chopping method for nulling interferometry," in Interferometry in Space. Proceedings of the SPIE, Volume 4852, M. Shao, ed., pp. 431442, Feb. 2003.

18. E. Serabyn, "An overview of the Keck Interferometer Nuller," in ESA SP-539: Earths: DARWIN/TPF and the Search for Extrasolar Terrestrial Planets, M. Fridlund, T. Henning, and H. Lacoste, eds., pp. 91-98, Oct. 2003.

19. L. L. D'Arcio, A. L. Karlsson, and P. A. Gondoin, "Use of OPD modulation techniques in nulling interferometry," in New Frontiers in Stellar Interferometry, Proceedings of SPIE Volume 5491, W. A. Traub, ed., pp. $851-+$, Oct. 2004

20. C. Hanot, "Investigation of a down scoped version of the darwin mission," Master's thesis, Liège University, Liège, Belgium, 2005.

21. T. Kelsall, J. L. Weiland, B. A. Franz, W. T. Reach, R. G. Arendt, E. Dwek, H. T. Freudenreich, M. G. Hauser, S. H. Moseley, N. P. Odegard, R. F. Silverberg, and E. L. Wright, "The COBE Diffuse Infrared Background Experiment Search for the Cosmic Infrared Background. II. Model of the Interplanetary Dust Cloud," ApJ 508, pp. 44-73, Nov. 1998.

22. C. Leinert, S. Bowyer, L. K. Haikala, M. S. Hanner, M. G. Hauser, A.-C. Levasseur-Regourd, I. Mann, K. Mattila, W. T. Reach, W. Schlosser, H. J. Staude, G. N. Toller, J. L. Weiland, J. L. Weinberg, and A. N. Witt, "The 1997 reference of diffuse night sky brightness," AEAS 127, pp. 1-99, Jan. 1998.

23. I. Baraffe, G. Chabrier, T. S. Barman, F. Allard, and P. H. Hauschildt, "Evolutionary models for cool brown dwarfs and extrasolar giant planets. The case of HD 209458," A\&̈A 402, pp. 701-712, May 2003.

24. S. Ida and D. N. C. Lin, "Toward a Deterministic Model of Planetary Formation. I. A Desert in the Mass and Semimajor Axis Distributions of Extrasolar Planets," Astrophysical Journal of Physics 604, pp. 388-413, Mar. 2004.

25. R. den Hartog, "The DARWINsim science simulator," Tech. Rep. Issue 1, ESA (SCI-A/297), May 2005.

26. J. F. Kasting, D. P. Whitmire, and R. T. Reynolds, "Habitable Zones around Main Sequence Stars," Icarus 101, pp. 108-128, Jan. 1993.

27. J. Scalo, L. Kaltenegger, A. G. Segura, M. Fridlund, I. Ribas, Y. N. Kulikov, J. L. Grenfell, H. Rauer, P. Odert, M. Leitzinger, F. Selsis, M. L. Khodachenko, C. Eiroa, J. Kasting, and H. Lammer, "M Stars as Targets for Terrestrial Exoplanet Searches And Biosignature Detection," Astrobiology 7, pp. 85-166, Feb. 2007.

28. D. Defrère, "Performance study of spaced-based infrared nulling interferometers," Master's thesis, Liège University, Liège, Belgium, 2007. 